\title{
Performance Evaluation of Outpatient Department Waiting Line System in a City Hospital in Nigeria
}

\author{
ADAJI, I; *2 LAWAL, A; ${ }^{3}$ ABDULLAHI, A; ${ }^{4}$ ABDULKADIR, A \\ ${ }^{1,2}$ Mathematics Department, Federal University of Technology, Minna, Nigeria \\ ${ }^{3}$ Department of Mathematics, Faculty of Science, Air Force Institute of Technology, Kaduna, Nigeria \\ ${ }^{4}$ Department of Mathematical Science, Ibrahim Badamasi Babagida University, Lapai, Nigeria \\ *Corresponding Author Email: lawal.adamu@futminna.edu.ng
}

\begin{abstract}
In this paper, performance evaluation of outpatient department waiting line system in a city hospital in Nigeria has been studied using a multiple server queuing model. The relevant data used in the research were collected for a period of four weeks through direct observations and interviews. The results of the research showed that with Two Doctors for the morning session patients spent an average of 1.0233 hours in the system, out of which 0.932 hours are spent on the queue with $95.45 \%$ Doctor Utilization. When the numbers of the Doctors were increased to 3, 4 and 5, it was found that a patient will spend an average of 0.125 hours, 0.0975 hours and 0.0924 hours in the system respectively. In each of the 3, 4 and 5 Doctors increased, the system will have $63.64 \%, 47.73 \%$ and $38.18 \%$ Doctors utilization respectively. With one Doctor for the evening session, the system has $54.55 \%$ Doctor Utilization and patient spent 0.2 hours in the system. The overall results showed that there is need to increase the number of Doctors to 3 or 4 for the morning session to achieved optimal service delivery while one Doctor in the evening session should be maintained. The results from the research could serves as important information to the management of the hospital for better services delivery.
\end{abstract}

\section{DOI:https://dx.doi.org/10.4314/jasem.v25i1.9}

Copyright: Copyright $(\mathrm{C} 2021$ Adaji et al. This is an open access article distributed under the Creative Commons Attribution License (CCL), which permits unrestricted use, distribution, and reproduction in any medium, provided the original work is properly cited.

Dates: Received: 02 October 2020; Revised: 26 November 2020; Accepted: 12 December 2020

Keywords: Doctors, Patient, Doctor Utilization, Queuing model, Evaluation

Queuing theory is the mathematical study of waiting lines (Onaja et al., 2018). Queuing theory was first analyzed by A.K. Erlang a Danish Engineer in 1913 in the context of telephone facilities. He was experimenting with the fluctuating demand for telephone facilities and its effect on automatic dialling equipment at the Copenhagen telephone System (Gupta and Hira, 1979). Queues are mostly formed when customers (arrivals) demanding service have to wait because their number exceeds the number of servers available or the facility does not work efficiently or takes more than the time prescribed to service a customer (Asogwa et al., 2019). Queues are usually seen at bus stops, hospitals, filling stations, bank counters and so on. In the application of queuing models in the hospitals, patients are considered as customers and different units of the hospital such as outpatient department, emergency unit, pharmacy, laboratory or diagnostic imaging are referred as services facilities. Waiting time depends on the number of patients on the queue, the number of health personnel serving the patients on the queue, and the amount of service time for each individual patient. The study of queuing systems has often been concerned with the busy period and the waiting time, because they play a very significant role in the understanding of various queuing systems and their management. Queuing models in general, are especially useful when predicting performance measures from the systems they model, such as the empty system probability, expected number of customers in the systems, expected number of customers on the queue and expected waiting time in the system (Cruz et al., 2020).It is well known that keeping patients for so long on queues has tendency of worsening their health situation or led to patients' dissatisfactions as a results of waiting cost incurred and providing too much service capacity may lead to excessive costs on the path of the hospital management. Many researchers have tried to help several healthcare providers with information that will assist them in best decision making, among them are: Singh (2011) had developed a model for evaluating the impact of bed assignment policies on utilization, waiting time, and the probability of turning away patients. Sundampandian (2009) used queuing theory in pharmacy application with particular attention to improving customer satisfaction. Customer satisfaction was improved by predicting and reducing waiting times and adjusting staffing. Singh (2006) considered a pharmacy queuing 
system with pre-emptive service priority discipline where the arrival of a prescription order suspends the processing of lower priority prescriptions. A successful application of queuing system in hospital management has also been reported in (John, 2010;Olaniyi, 2004; Kembe and Onah, 2012; Lakshmi, and Sivakumar, 2013).The aim of this research is to investigate the performance level of the queuing system in the outpatient department of National Health Insurance Scheme Unit in General Hospital Minna, Niger state, Nigeria with a view to providing the management of the hospital with information that could be used to provide optimal service delivery to her patients without incurring unnecessary cost.

\section{MATERIALS AND METHODS}

Study Area and Data Source: The research was carried out in General Hospital Minna, Niger state, Nigeria. Minna General Hospital is a state owned hospital that affords the residents of Minna and the environment to access medical services at a subsides rate. National Health Insurance Scheme (NHIS) is a corporate body established under Act 35 of 1999 Constitution by the federal government of Nigeria, under the government of former president Oludegun Obasajo (GCFR), in order to improve the healthcare of all Nigerians at an affordable rate, through various prepayment systems. The NHIS unit of the General Hospital Minna, is one of the outpatient departments in the hospital that offers medical services for registered members of the scheme.

The data used in this research were collected from National Health Insurance Scheme (NHIS) unit of the General Hospital Minna. The methods employed during data collection were direct observation and personal interview administered by the researchers. Data were collected for four weeks. The NHIS unit is opened from 8Am till 9Pm everyday. The following assumptions were made for queuing system at the NHIS unit of the general hospital Minna. They are:

1. Arrivals follow a Poisson probability distribution at an average rate of $\lambda$ patients per unit of time.

2. The queue discipline is First-Come, FirstServed (FCFS) basis by any of the servers. There is no priority classification for any arrival.

3. Service times are distributed exponentially, with an average of $\mu$ patients per unit of time.

4. There is no limit to the number of the queue (infinite).

5. The service providers are working at their full capacity.
6. The average arrival rate is greater than average service rate.

7. Servers here represent only doctors but no other medical personnel's.

8. Service rate is independent of line length.

9. Balking and Reneging is not considered in the system.

The M/M/S Model: The model adopted in this work is the $(\mathrm{M} / \mathrm{M} / \mathrm{S}):(\infty / \mathrm{FCFS})$ - Multi-server Queuing Model. For this queuing system, it is assumed that the arrivals follow a Poisson probability distribution at an average of $\lambda$ customers (patients) per unit of time. It is also assumed that they are served on a first-come, firstserved basis by any of the servers (in this case is doctors). The service times are distributed exponentially, with an average of $\mu$ customers (patients) per unit of time and number of servers S. If there are $\mathrm{n}$ customers in the queuing system at any point in time, then the following two cases may arise: (i) If $\mathrm{n}<\mathrm{S}$, (number of patients in the system is less than the number of servers), then there will be no queue. However, $(\mathrm{S}-\mathrm{n})$ number of servers will not be busy. The combined service rate will then be $\mu_{\mathrm{n}}<\mathrm{n} \mu ; \mathrm{n}$ $<\mathrm{S}$ (ii) If $\mathrm{n} \geq \mathrm{S}$, (number of patients in the system is more than or equal to the number of servers) then all servers will be busy and the maximum number of patients in the queue will be

$(n-S)$. The combined service rate will be $\mu_{n} \geq S \mu ; n \geq$ S.

The following are the queuing model equations to be used.

The probability of having $\mathrm{n}$ patients in the system is given by

$$
\begin{aligned}
& P_{n}= \begin{cases}\left(\frac{\rho^{n}}{n !}\right) p_{0} & n<s \\
\frac{\rho^{n}}{\left(s ! s^{n-s}\right) p_{n}} & n \geq s ; \rho=\frac{\lambda}{s \mu}\end{cases} \\
& P_{0}=\left(\left(\sum_{n=0}^{s-1} \frac{1}{n !}\left(\frac{\lambda}{\mu}\right)^{n}\right)+\frac{1}{s !} \frac{\left(\frac{\lambda}{\mu}\right)^{s} s \mu}{s \mu-\lambda}\right)^{-1}
\end{aligned}
$$

$P_{0}$ is the probability that there are no patients in the system.

$$
L q=\left(\frac{1}{(S-1) !}\left(\frac{\lambda}{\mu}\right)^{S} \frac{\mu \lambda}{(S \mu-\lambda)^{2}}\right) P_{0}
$$

$L q$ is the expected number of the patients waiting on the queue (length of line), this is the number of patients waiting on the queue to be serve by a Doctor. 


$$
L s=\left(\frac{1}{(S-1) !}\left(\frac{\lambda}{\mu}\right)^{s} \frac{\mu \lambda}{(S \mu-\lambda)^{2}}\right) P_{0}+\frac{\lambda}{\mu}(4)
$$

$L s$ is the expected number of patients in the system

$W q=\frac{\left(\frac{1}{(S-1) !}\left(\frac{\lambda}{\mu}\right)^{s} \frac{\mu \lambda}{(S \mu-\lambda)^{2}}\right) P_{0}}{\lambda}$

$W q$ is the expected waiting time of patients in the queue, this is the time that the patient spend before being seen by a Doctor.

$W s=\frac{\left(\frac{1}{(S-1) !}\left(\frac{\lambda}{\mu}\right)^{s} \frac{\mu \lambda}{(S \mu-\lambda)^{2}}\right) P_{0}+\frac{\lambda}{\mu}}{\lambda}(6)$

$W s$ is the average time a patients spends in the system, this is the time that the patient spent in the hospital (waiting in queue and seeing the Doctor).

$$
\rho=\frac{\lambda}{S \mu}
$$

$\rho$ is utilization factor that is the fraction of time Doctors are busy

Where, $\lambda$ is the arrival rate of patients per unit time, $\mu$ is the service rate per unit time, $\mathrm{S}$ is the number of
Doctors, $\mathrm{n}$ is the number of patients in the system, $P_{0}$ $=$ the probability that there are no or zero patients in the system.

\section{RESULTS AND DISCUSSION}

We used MAPLE software to compute the performance measures of the multi-server queuing system at the National Health Insurance Scheme (NHIS) unit of the General Hospital Minna for the morning session (8:00Am - 1:00Pm), we obtained our arrival rate $(\lambda)$ to be 21 patients per hour and our service rate $(\mu)$ to be 11 patients per hour from our raw data obtained, and number of servers to be $(S)=2$.

From equation (2), the probability that there are no patients in the system or the system is idle is given by

$$
\begin{aligned}
& P_{0}=\left(\left(\sum_{n=0}^{s-1} \frac{1}{n !}\left(\frac{\lambda}{\mu}\right)^{n}\right)+\frac{1}{s !} \frac{\left(\frac{\lambda}{\mu}\right)^{s} s \mu}{s \mu-\lambda}\right)^{-1} \\
& =\left(\left(\frac{1}{0 !}\left(\frac{21}{11}\right)^{0}\right)+\frac{1}{2 !} \frac{\left(\frac{21}{11}\right)^{2} 2 \times 11-21}{2 \times 11}\right)^{-1}+\left(\left(\frac{1}{1 !}\left(\frac{21}{11}\right)^{1}\right)+\frac{1}{2 !} \frac{\left(\frac{21}{11}\right)^{2} \times 2 \times 11}{2 \times 11-21}\right)^{-1}=0.0233
\end{aligned}
$$

From equation (3), The mean length of patients on the queue is given as

$$
L q=\left(\frac{1}{(S-1) !}\left(\frac{\lambda}{\mu}\right)^{s} \frac{\mu \lambda}{(S \mu-\lambda)^{2}}\right) P_{0}
$$

$L q=\left(\frac{1}{(2-1) !}\left(\frac{21}{11}\right)^{2} \times \frac{11 \times 21}{(2 \times 11-21)^{2}}\right)\left(\left(\frac{1}{0 !}\left(\frac{21}{11}\right)^{0}\right)+\frac{1}{2 !} \frac{\left(\frac{21}{11}\right)^{2} \times 2 \times 11}{2 \times 11-21}\right)^{-1}+\left(\left(\frac{1}{1 !}\left(\frac{21}{11}\right)^{1}\right)+\frac{1}{2 !} \frac{\left(\frac{21}{11}\right)^{2} \times 2 \times 11}{2 \times 11-21}\right)^{-1}$
From equation (4),

The mean length of patients in the system is given as

$$
\begin{aligned}
& L s=L q+\frac{\lambda}{\mu} \\
& L s=\left(\frac{1}{(S-1) !}\left(\frac{\lambda}{\mu}\right)^{s} \frac{\mu \lambda}{(S \mu-\lambda)^{2}}\right) P_{0}+\frac{\lambda}{\mu} \\
& L s=\left(\frac{1}{(2-1) !}\left(\frac{21}{11}\right)^{2} \times \frac{11 \times 21}{(2 \times 11-21)^{2}}\right) 0.0233+\frac{21}{11}=21.4882
\end{aligned}
$$

From equation (5), the waiting time of patients on the queue is given as

$$
\begin{aligned}
& W q=\frac{\left(\frac{1}{(S-1) !}\left(\frac{\lambda}{\mu}\right)^{2} \frac{\mu \lambda}{(S \mu-\lambda)^{2}}\right) P_{0}}{\lambda} \\
& W q=\frac{\left(\frac{1}{(2-1) !}\left(\frac{21}{11}\right)^{2} \times \frac{11 \times 21}{(2 \times 11-21)^{2}}\right)(0.0233)}{21}=0.9323 \text { hours }
\end{aligned}
$$

A patient spent 55.938minutes on queue From equation (6),

The waiting time of patients in the system is given as

$$
\begin{aligned}
& W s=\frac{\left(\frac{1}{(S-1) !}\left(\frac{\lambda}{\mu}\right)^{S} \frac{\mu \lambda}{(S \mu-\lambda)^{2}}\right) P_{0}+\frac{\lambda}{\mu}}{\lambda} \\
& W S=\frac{\left(\frac{1}{(2-1) !}\left(\frac{21}{11}\right)^{2} \times \frac{11 \times 21}{(2 \times 11-21)^{2}}\right)(0.0233)+\frac{21}{11}}{21}=1.0233 \text { hours }
\end{aligned}
$$

A patient spent 61.398 minutes in the system From equation (7),

The utilization factor is given by

$$
\begin{aligned}
& \rho=\frac{\lambda}{S \mu} \\
& \rho=\frac{21}{2 \times 11} \times 100=95.45 \%
\end{aligned}
$$


The summary of the computed performance measure for morning session of the multi-server queuing model at NHIS unit in General Hospital, Minna is given in the tables 1 and 2.From Table 1, it can be observed that with two Doctors in the unit for the morning session, the system has $95.45 \%$ utilization factor. A Patient spent an average of 1.0233 hours (61.4 minutes) in the system out of which 0.932 hours ( 55.9 minutes) is spent on queue. This waiting time is certainly unfavourable to them, to this end we increased the number of Doctors to 3, 4, and 5 then make computation for each case. This is to provide the hospital management with information for better service delivery. The results from the computation is presented in Table 2. From Table2, it can be seen that when the numbers of the Doctors were increased to 3, 4 and 5, the utilization factors are $63.64 \%, 47.73 \%$ and $38.18 \%$ respectively while with the 3, 4 and 5 Doctors, A patients will spent an average of 0.1245 hours 7.5 minutes), 0.0975 hours $(5.9$ minutes $)$ and 0.0924 hours(5.5minutes) in the system respectively.

Table 1.Performance Measure of the Queuing System, for the Morning Session at NHIS Unit of the General Hospital, Minna.

\begin{tabular}{ll}
\hline No. of servers & 2 Doctors \\
\hline Arrival rate $(\boldsymbol{\lambda})$ & 21 \\
Service rate $(\boldsymbol{\mu})$ & 11 \\
System utilization $(\boldsymbol{\rho})$ & $95.45 \%$ \\
$\mathbf{L}_{\mathbf{S}}$ & 21.4884 \\
$\mathbf{L}_{\mathbf{q}}$ & 19.5793 \\
$\mathbf{W}_{\mathbf{S}}$ in hours & 1.0233 \\
$\mathbf{W}_{\mathbf{q}}$ in hours & 0.9323 \\
$\mathbf{P}_{\mathbf{0}}$ & 0.0233 \\
\hline
\end{tabular}

Table 2. Performance Measure of the Queuing System when the numbers of Doctors were increased to 3, 4 and 5 .

\begin{tabular}{lllll}
\hline No. of servers & 2 Doctors & 3 Doctors & 4 Doctors & 5 Doctors \\
\hline Arrival rate $(\boldsymbol{\lambda})$ & 21 & 21 & 21 & 21 \\
Service rate $(\boldsymbol{\mu})$ & 11 & 11 & 11 & 11 \\
System utilization $(\boldsymbol{\rho})$ & $95.45 \%$ & $63.64 \%$ & $47.73 \%$ & $38.18 \%$ \\
$\mathbf{L}_{\mathbf{S}}$ & 21.4884 & 2.6137 & 2.0481 & 1.9402 \\
$\mathbf{L}_{\mathbf{q}}$ & 19.5793 & 0.7046 & 0.1391 & 0.0311 \\
$\mathbf{W}_{\mathbf{S}}$ in hours & 1.0233 & 0.1245 & 0.0975 & 0.0924 \\
$\mathbf{W}_{\mathbf{q}}$ in hours & 0.9323 & 0.0336 & 0.0066 & 0.0015 \\
$\mathbf{P}_{\mathbf{0}}$ & 0.0233 & 0.1263 & 0.1439 & 0.1474 \\
\hline
\end{tabular}

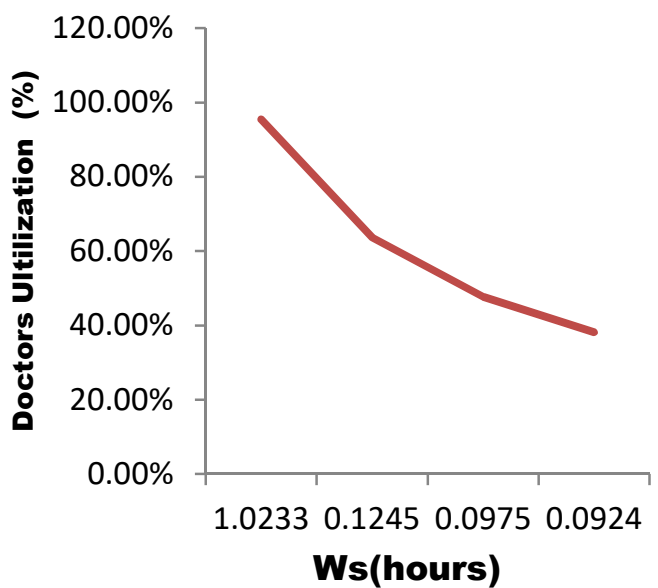

Fig 1: Time spent in the system with respect to Doctors utilization

Similarly, we compute the performance measures of the queuing system for the evening session $(4: 00 \mathrm{Pm}-$ 9:00Pm) as follows: the arrival rate $(\lambda)$ was found to be 6 patients per hour and service rate $(\mu)$ was 11 patients per hour and number of server is $(S)=1$ ( that is number of Doctor for the evening session). From equation (2). The probability that there are no patients in the queue or the system is idle is given by

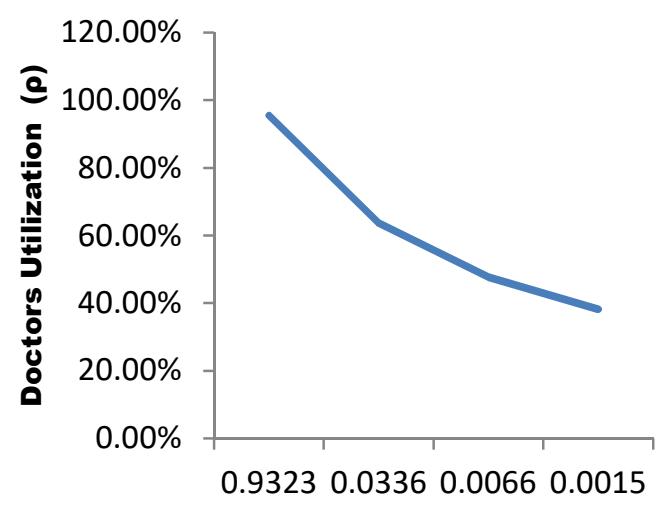

Wq in hours

Fig 2: waiting time on the queue with respect to Doctors utilization

$$
\begin{aligned}
& P_{0}=\left(\left(\sum_{n=0}^{S-1} \frac{1}{n !}\left(\frac{\lambda}{\mu}\right)^{n}\right)+\frac{1}{s !} \frac{\left(\frac{\lambda}{\mu}\right)^{S} s \mu}{s \mu-\lambda}\right)^{-1} \\
& \left.=\left(\frac{1}{0 !}\left(\frac{6}{11}\right)^{0}\right)+\frac{1}{1 !} \frac{\left(\frac{6}{11}\right)^{1} \times 1 \times 11}{1 \times 11-6}\right)^{-1}=0.4545
\end{aligned}
$$

From equation (3), the mean length of patients on the queue is given as 


$$
\begin{aligned}
& L q=\left(\frac{1}{(S-1) !}\left(\frac{\lambda}{\mu}\right)^{s} \frac{\mu \lambda}{(S \mu-\lambda)^{2}}\right) P_{0} \\
& L q=\left(\frac{1}{(1-1) !}\left(\frac{6}{11}\right)^{1} \times \frac{11 \times 6}{(6 \times 11-6)^{2}}\right) 0.4545=0.6545
\end{aligned}
$$

From equation (4), the mean length of patients in the system is given as

$$
\begin{aligned}
& L s=L q+\frac{\lambda}{\mu} \\
& L s=\left(\frac{1}{(S-1) !}\left(\frac{\lambda}{\mu}\right)^{s} \frac{\mu \lambda}{(S \mu-\lambda)^{2}}\right) P_{0}+\frac{\lambda}{\mu} \\
& L s=\left(\frac{1}{(1-1) !}\left(\frac{6}{11}\right)^{1} \times \frac{11 \times 6}{(1 \times 11-6)^{2}}\right) 0.4545+\frac{6}{11}=1.2000
\end{aligned}
$$

From equation (5), the waiting time of patients on the queue is given as

$$
\begin{aligned}
& W q=\frac{\left(\frac{1}{(S-1) !}\left(\frac{\lambda}{\mu}\right)^{S} \frac{\mu \lambda}{(S \mu-\lambda)^{2}}\right) P_{0}}{\lambda} \\
& W q=\frac{\left(\frac{1}{(1-1) !}\left(\frac{6}{11}\right)^{1} \times \frac{11 \times 6}{(1 \times 11-6)^{2}}\right) 0.4545}{6}=0.1091 \text { hours }
\end{aligned}
$$

A patient spent 6.546 minutes on queue.

From equation (6), The waiting time of patients in the system is given as

$$
\begin{aligned}
& W S=\frac{\left(\frac{1}{(S-1) !}\left(\frac{\lambda}{\mu}\right)^{S} \frac{\mu \lambda}{(S \mu-\lambda)^{2}}\right) P_{0}+\frac{\lambda}{\mu}}{\lambda} \\
& W S=\frac{\left(\frac{1}{(1-1) !}\left(\frac{6}{11}\right)^{1} \times \frac{11 \times 6}{(1 \times 11-6)^{2}}\right) 0.4545+\frac{6}{11}}{6}=0.20
\end{aligned}
$$

session, the system has $54.55 \%$ utilization factor and a patient spent an average of 0.2 hours (12Munites) in the system out of which $0.1091(6.5$ minutes) is spent on the queue. With this waiting time in the system, there may not be need for additional Doctor(s) in the unit for the evening session. However, we have computed performance measure for 2 and 3 Doctors for more information

Table 3.Performance Measure for the evening session with one Doctor in the Unit.

\begin{tabular}{ll}
\hline No. of Servers & 1 Doctor \\
\hline Arrival rate $(\boldsymbol{\lambda})$ & 6 \\
Service rate $(\boldsymbol{\mu})$ & 11 \\
System utilization $\boldsymbol{\rho}$ & $54.55 \%$ \\
$\mathbf{L}_{\mathbf{S}}$ & 1.2000 \\
$\mathbf{L}_{\mathrm{q}}$ & 0.6545 \\
$\mathbf{W}_{\mathbf{s}}$ in hours & 0.2000 \\
$\mathbf{W}_{\mathrm{q}}$ in hours & 0.1091 \\
$\mathbf{P}_{\mathbf{0}}$ & 0.4545 \\
\hline
\end{tabular}

Table 4.Performance Measure for the evening session when the numbers of the Doctor were increased to 2 and 3 .

\begin{tabular}{llll}
\hline No. of Servers & 1 Doctor & 2 Doctors & 3 Doctors \\
\hline Arrival rate $(\boldsymbol{\lambda})$ & 6 & 6 & 6 \\
Service rate $(\boldsymbol{\mu})$ & 11 & 11 & 11 \\
System utilization $\boldsymbol{\rho}$ & $54.55 \%$ & $27.27 \%$ & $18.18 \%$ \\
$\mathbf{L}_{\mathbf{S}}$ & 1.2000 & 0.5893 & 0.5497 \\
$\mathbf{L}_{\mathbf{q}}$ & 0.6545 & 0.0438 & 0.0043 \\
$\mathbf{W}_{\mathbf{S}}$ in hours & 0.2000 & 0.0982 & 0.0916 \\
$\mathbf{W}_{\mathbf{q}}$ in hours & 0.1091 & 0.0073 & 0.0007 \\
$\mathbf{P}_{\mathbf{0}}$ & 0.4545 & 0.5714 & 0.5789 \\
\hline
\end{tabular}

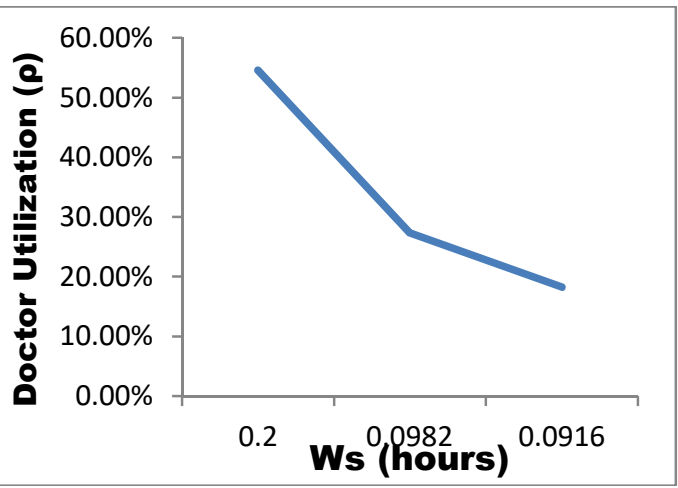

Fig 3: Time spent in the system with respect to Doctor Utilization

A patient spent 12 minutes in the system

From equation (7), the utilization factor is given by

$$
\begin{aligned}
& \rho=\frac{\lambda}{S \mu} \\
& \rho=\frac{6}{1 \times 11} \times 100=54.55 \%
\end{aligned}
$$

The summary of the computed performance measure for evening session of the multi-server queuing model at NHIS unit in General Hospital, Minna is given in the tables 3 and 5. With one Doctor in the evening

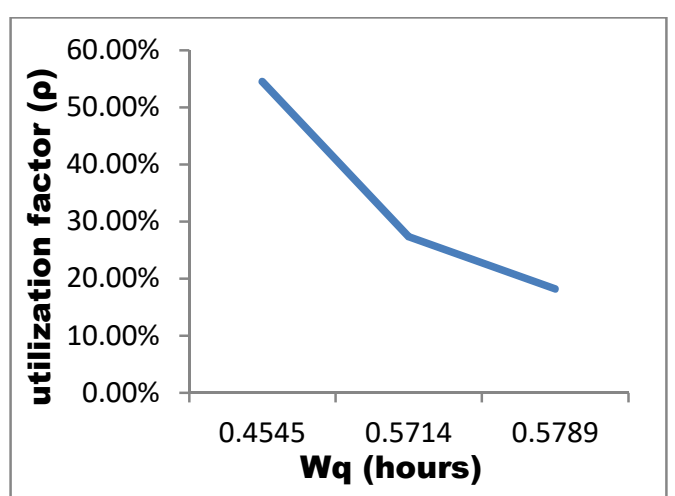

Fig 4: Waiting time on the queue with respect to Doctor utilization. 
Conclusion: The performance level of NHIS unit outpatient department in General hospital Minna has been effectively investigated using a multiple server queuing model. The overall results showed that there is need to increase the number of Doctors to Three or Four for the morning session to achieved optimal service delivery while one Doctor in the evening session should be maintained. The results from the research could serves as important information to the management of the hospital for better services delivery.

\section{REFERENCES}

Asogwa, O.C; Eze $\sigma$, C.M; Edeaja, MT (2019).On the Application of Queuing Model in Nigeria Banking Sector: A Case Study of Zenith Bank Plc, Abakaliki Branch. Global J. Sci. Frontier Res. Math. Decision Sci. 19(2) 51-58

Cruz, F.R.B; Quinino, R.C; Ho; L.L (2020).Control Charts for Traffic Intensity Monitoring of Markovian Multi-server Queues. Reliab. Eng.

Gupta, P.K; Hira, D.S (1979). Operations Research. Rajendra Ravindra Printers. New Delhi.

John, K.O. (2010). Queuing Theory and Patient Satisfaction. An overview of Terminology and Application in Ante-natal Care Unit. Bull. Petrol. Gas University of Ploiesti. 61: 1-10.

Kembe, M. M,Onah, E.S, lorkegh and S (2012) A Study of Waiting and Service cost of a MultiServer Queuing Model in a Specialist Hospital" Inter. J. Sci. Tech. Res. 1 (8) 19-23.
Lakshmi, C; Sivakumar, A.I. (2013.).Application of queuing theory in health care: A literature review. Oper. Res. Health Care, no. 2: 25-39.

Olaniyi, (2004).Waiting for Orthopaedic surgery.factors associated with waiting time and customers $^{\text {ce }}$ opinion. Inter. J. Quality in Healthcare. 17: 133-140.

Onoja, A.A; O.L Babasola, O.L; Edwin Moyo, Viona Ojiambo (2018). The Application of Queuing Analysis in modeling Optimal Service level. Inter. J. Sci. Eng. Res. 9(1) 184-194

Singh, V (2006).Use of Queuing Models in Health care. Department of health policy and management. University of Arkansas for medical Sciences.

Singh, V. (2011). Use of Queuing Models in Health Care, Department of Health Policy and Management, University of Arkanses for medical science. Inter. J. Comp. Bus. Res.1-2.

Sundarapandian, V. (2009).Queuing Theory. Probability, Statistics and Queuing Theory. PHI Learning, First edition. New Delhi. 